\title{
Extension of some edge graph problems: standard and parameterized complexity
}

\author{
Katrin Casel ${ }^{1,2}$, Henning Fernau ${ }^{2}$, Mehdi Khosravian Ghadikolaei ${ }^{3}$, \\ Jérôme Monnot ${ }^{3}$, and Florian Sikora ${ }^{3}$ \\ 1 Hasso Plattner Institute, University of Potsdam, 14482 Potsdam, Germany \\ 2 Universität Trier, Fachbereich 4, Informatikwissenschaften, \\ 54286 Trier, Germany, \{casel,fernau\}@informatik.uni-trier.de \\ 3 Université Paris-Dauphine, PSL University, CNRS, LAMSADE, 75016 Paris, \\ France, \{mehdi.khosravian-ghadikolaei, jerome.monnot, \\ florian.sikora\}@lamsade.dauphine.fr
}

\begin{abstract}
We consider extension variants of some edge optimization problems in graphs containing the classical Edge Cover, Matching, and Edge Dominating Set problems. Given a graph $G=(V, E)$ and an edge set $U \subseteq E$, it is asked whether there exists an inclusion-wise minimal (resp., maximal) feasible solution $E^{\prime}$ which satisfies a given property, for instance, being an edge dominating set (resp., a matching) and containing the forced edge set $U$ (resp., avoiding any edges from the forbidden edge set $E \backslash U$ ). We present hardness results for these problems, for restricted instances such as bipartite or planar graphs. We counterbalance these negative results with parameterized complexity results. We also consider the price of extension, a natural optimization problem variant of extension problems, leading to some approximation results.
\end{abstract}

Keywords: Extension problems $\cdot$ Edge Cover · Matching $\cdot$ Edge Domination · NP-completeness · Parameterized Complexity · Approximation

\section{Introduction}

We consider extension problems related to several classical edge optimization problems in graphs, namely Edge Cover, Maximum Matching and EdGe Dominating SET. Informally, in an extension version of an edge optimization problem, one is given a graph $G=(V, E)$ as well as a subset of edges $U \subseteq E$, and the goal is to extend $U$ to a minimal (or maximal) solution (if possible).

Such variants of problems are interesting for efficient enumeration algorithms or branching algorithms (see more examples of applications in [11]).

Related work Extension versions have been studied for classical optimization problems, for example, the minimal extension of 3-HitTing SET [9], minimal Dominating Set $[2,8]$ or Vertex Cover [1]. Extensions show up quite naturally in quite a number of situations. For instance, when running a search tree algorithm, usually parts of the constructed solution are fixed. It is highly desirable to be able to prune branches of the search tree as early as possible. Hence, 
it would be very nice to tell efficiently if such a solution part can be extended to a valid (minimal) solution. When trying to enumerate all minimal solutions, the same type of problem arises and has known applications in so-called flashlight algorithms [24]. Another type of area where extension problems show up is linked to Latin squares [13] (and similar combinatorial questions), or also coloring extensions in graphs [7]. In a recent paper, we investigated the complexity of extension versions of Vertex Cover and IndePendent Set, i.e., classical vertex graph problems [12], and we give a first systematic study of this type of problems in [11], providing quite a number of different examples of extension problems. For extension variants of automata-related problems, see [17].

Organization of the paper After giving some definitions in Section 2, we prove that generalization of these problems remain NP-complete, even in bipartite graphs of bounded degree and with some constraints on the forced set of edges. Having a planar embedding does not help much either, as we show in Section 4 that these problems remain hard on subcubic bipartite planar graphs. Motivated by these negative results, we study the parameterized complexity of these problems in Section 5 and the approximability of a natural optimization version in Section 6. Due to lack of space the proofs of statements marked with $(*)$ are deferred to the full version of the paper.

\section{Definitions}

Graph definitions We consider simple undirected graphs only, to which we refer to as graphs. Let $G=(V, E)$ be a graph and $S \subseteq V ; N_{G}(S)=\{v \in V: \exists u \in$ $S, v u \in E$ \} denotes the neighborhood of $S$ in $G$ and $N_{G}[S]=S \cup N_{G}(S)$ denotes the closed neighborhood of $S$. For singleton sets $S=\{s\}$, we simply write $N_{G}(s)$ or $N_{G}[s]$, even omitting $G$ if clear from context. The cardinality of $N_{G}(s)$ is called degree of $s$, denoted $d_{G}(s)$. If 3 upper-bounds the degree of all vertices, we speak of subcubic graphs. For a subset of edges $S, V(S)$ denotes the vertices incident to $S$. A vertex set $S$ induces the graph $G[S]$ with vertex set $S$ and $e \in E$ being an edge in $G[S]$ iff both endpoints of $e$ are in $S$. If $S \subseteq E$ is an edge set, then $\bar{S}=E \backslash S$, edge set $S$ induces the graph $G[V(S)]$, while $G_{S}=(V, S)$ denotes the partial graph induced by $S$; in particular, $G_{\bar{S}}=(V, E \backslash S)$.

A vertex set $S$ is independent if $S$ is a set of pairwise non-adjacent vertices. An edge set $S$ is called an edge cover if the partial graph $G_{S}$ is spanning and it is a matching if $S$ is a set of pairwise non-adjacent edges. An edge set $S$ is minimal (resp., maximal) with respect to a graph property if $S$ satisfies the graph property and any proper subset $S^{\prime} \subset S$ of $S$ (resp., any proper superset $S^{\prime} \supset S$ of $S$ ) does not satisfy the graph property. A graph $G=(L \cup R, E)$ is called bipartite if its vertex set decomposes into two independent sets $L$ and $R$. The line graph $L(G)=\left(V^{\prime}, E^{\prime}\right)$ of a graph $G=(V, E)$ is a simple graph where each vertex of $L(G)$ represents an edge of $G$ and two vertices of $L(G)$ are adjacent if and only if their corresponding edges share a common vertex in $G$. Hence, it is exactly the intersection graph of the edges of $G$. It is well known the class of line graphs is a subclass of claw-free graphs (i.e., without $K_{1,3}$ as induced subgraph). 
Problem definitions Let $G=(V, E)$ be a graph where the minimum degree is at least $r \geq 1$. We assume $r$ is a fixed constant (but all results given here hold even if $r$ depends on the graph). An $r$-degree constrained partial subgraph is defined as an edge subset $S \subseteq E$ such that none of the vertices in $V$ is incident to more than $r$ edges in $S$. The problem of finding such a set $S$ of size at least $k$ is termed $r$-DCPS. An $r$-degree edge-cover is defined as a subset of edges such that each vertex of $G$ is incident to at least $r \geq 1$ distinct edges $e \in S$, leading to the decision problem $r$-EC, determining if such a set of size at most $k$ exists. For the particular cases of $r=1,1$-DCPS corresponds to the famous MATCHING problem and 1-EC is also known as the EDGE COvER problem.

The optimization problem associated to $r$-DCPS, denoted MAX $r$-DCPS, consists of finding an edge subset $E^{\prime}$ of maximum cardinality that is a solution to $r$-DCPS. MAX $r$-DCPS is known to be solvable in polynomial time even for the edge weighted version (here, we want to maximize the weight of $E^{\prime}$ ) [19]. When additionally the constraint $r$ is not uniform and depends on each vertex (i.e., at most $b(v)=r_{v}$ edges incident to vertex $v$ ), MAX $r$-DCPS is usually known as Simple $b$-MATChing and remains solvable in polynomial time even for the edge-weighted version (Theorem 33.4, Chapter 33 of Volume A in [27]).

A well-studied optimization version of a generalization of $r$-EC, known as the Min Lower-upper-COVER Problem (MinLUCP), is the following. Given a graph $G=(V, E)$ and two functions $a, b: V \rightarrow \mathbb{N}$ such that for all $v \in V$, $0 \leq a(v) \leq b(v) \leq d_{G}(v)$, find a subset $M \subseteq E$ such that the partial graph $G_{M}=(V, M)$ induced by $M$ satisfies $a(v) \leq d_{G_{M}}(v) \leq b(v)$ (such a solution will be called a lower-upper-cover), minimizing its cardinality $|M|$ among all such solutions (if any). Hence, an $r$-EC solution corresponds to a lower-upper-cover with $a(v)=r$ and $b(v)=d_{G}(v)$ for every $v \in V$. MinLUCP is known to be solvable in polynomial time even for edge-weighted graphs (Theorem 35.2 in Chapter 35 of Volume A in [27]).

We are considering the following extension problems associated to $r$-DCPS and $r$-EC.

ExT $r$-DCPS

Input: A graph $G=(V, E)$ and $U \subseteq E$.

Question: Does there exist an edge set $S \subseteq E$ with $S \subseteq U$ such that the partial graph $G_{S}$ has maximum degree at most $r$ and is maximal in $G$ ?

\section{ExT $r$-EC}

Input: A graph $G=(V, E)$ and $U \subseteq E$.

Question: Does there exist an edge set $S \subseteq E$ with $S \supseteq U$ such that the partial graph $G_{S}$ has minimum degree at least $r$ and is minimal in $G$ ?

An $r$-edge dominating set $S \subseteq E$ of a simple graph $G=(V, E)$ is a set $S$ of edges such that for any edge $e \in E$ of $G$, at least $r$ edges of $S$ are incident to $e$ (by definition, an edge dominates itself one time). The Minimum $r$-EDGE Dominating SeT problem (Min $r$-EDS for short) consists in finding an $r$-edge dominating set of minimum size. Notice that there is a feasible solution if and only if $r \leq \min _{x y \in E}\left(d_{G}(x)+d_{G}(y)-1\right)$. Obviously, 1-EDS is the classical EDGE 
Dominating SET problem (EDS), which is NP-hard in general graphs (problem [GT2] in [20]). The generalization to $r$-EDS has been studied in $[3,4]$ (under the name $b$-EDS) from an approximation point of view. However, to the best of our knowledge, $r$-EDS for every $r \geq 2$ was not proved NP-hard so far. As associated extension problem, we formally study the following problem.

ExT $r$-EDS

Input: Given a simple graph $G=(V, E)$ and $U \subseteq E$.

Question: Is there a subset $S \subseteq E$ such that $U \subseteq S$ and $S$ is a minimal $r$-edge dominating set?

For an edge extension problem $\pi, \operatorname{ext}_{\pi}(G, U)$ denotes the set of extremal extensions of $U$ (i.e., minimal or maximal depending on the context). For a minimal version, $U$ corresponds to a subset of forced edges (i.e., each minimal solution has to contain $U$ ) while for a maximal version, $E \backslash U$ corresponds to a subset of forbidden edges (i.e., each maximal solution has to contain no edges from $E \backslash U)$. Sometimes, the set $\operatorname{ext}_{\pi}(G, U)$ is empty, which makes the question of the existence of such extensions interesting. Hence, for $\pi \in\{$ EXT $r$-DCPS,EXT $r$-EC,EXT $r$-EDS $\}$, the extension problems ask if $\operatorname{ext}_{\pi}(G, U) \neq \emptyset$. We call $|U|$ the standard parameter when considering these problems as parameterized. We may drop the subscript $\pi$ if clear from context.

\section{Complexity results}

The results given in this section are based on a reduction from 2-BALANCED 3 -SAT, $(3, B 2)$-SAT for short. An instance $(\mathcal{C}, \mathcal{X})$ of $(3, B 2)$-SAT is a set $\mathcal{C}$ of $\mathrm{CNF}$ clauses defined over a set $\mathcal{X}$ of Boolean variables such that each clause has exactly 3 literals and each variable appears exactly twice as a negative and twice as a positive literal in $\mathcal{C}$. The bipartite graph associated to $(\mathcal{C}, \mathcal{X})$ is $B P=(C \cup$ $X, E(B P))$, with $C=\left\{c_{1}, \ldots, c_{m}\right\}, X=\left\{x_{1}, \ldots, x_{n}\right\}$ and $E(B P)=\left\{c_{j} x_{i}: x_{i}\right.$ or $\neg x_{i}$ is a literal of $\left.c_{j}\right\}$. (3,B2)-SAT is NP-hard by [5, Theorem 1].

Theorem 1. (*) For every fixed $r \geq 1$, ExT $r$-DCPS is NP-complete in bipartite graphs with maximum degree $\max \{3, r+1\}$, even if $\bar{U}$ is an induced matching for $r \geq 2$ or an induced collection of paths of length at most 2 for $r=1$.

Proof. Let $r=1$. For the technical details for the case $r>1$, we refer to the long version of this paper. Consider an instance of (3, B2)-SAT with clauses $\mathcal{C}=\left\{c_{1}, \ldots, c_{m}\right\}$ and variables $\mathcal{X}=\left\{x_{1}, \ldots, x_{n}\right\}$. We build a bipartite graph $G=(V, E)$ of maximum degree 3 as follows:

- For each clause $c=x \vee y \vee z$, where $x, y, z$ are literals, introduce a subgraph $H(c)=\left(V_{c}, E_{c}\right)$ with 8 vertices and 9 edges. $V_{c}$ contains three specified vertices $x_{c}, y_{c}$ and $z_{c}$ corresponding to literals of the clause $c$. Moreover, $\bar{U}_{c}=\left\{x_{c} 1_{c}, y_{c} 2_{c}, z_{c} 3_{c}\right\}$ is a set of three forbidden edges included in $H(c)$. The gadget $H(c)$ is illustrated in the left part of Fig. 1. 


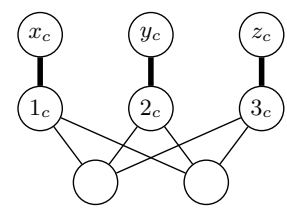

$H(c)$ for $c=x \vee y \vee z$

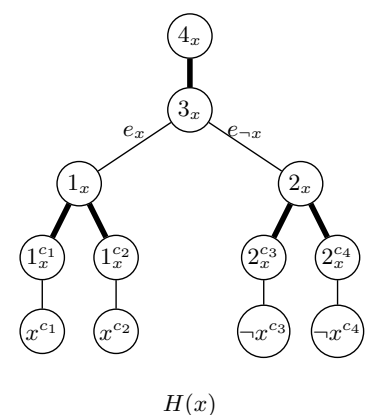

Fig. 1. The Gadgets $H(c)$ and $H(x)$. Edges not in $U$ are drawn as thicker lines.

- For each variable $x$, introduce 12 new vertices. They induce the subgraph $H(x)=\left(V_{x}, E_{x}\right)$ illustrated in Fig. 1. The vertex set $V_{x}$ contains four special vertices $x^{c_{1}}, x^{c_{2}}, \neg x^{c_{3}}$ and $\neg x^{c_{4}}$, where it is implicitly assumed that variable $x$ appears as a positive literal in clauses $c_{1}, c_{2}$ and as a negative literal in clauses $c_{3}, c_{4}$. Finally, there are two sets of free edges (non-forbidden edges): $F_{x}=\left\{e_{x}\right\} \cup\left\{2_{x}^{c_{3}} \neg x^{c_{3}}, 2_{x}^{c_{4}} \neg x^{c_{4}}\right\}$ and $F_{\neg x}=\left\{e_{\neg x}\right\} \cup\left\{1_{x}^{c_{1}} x^{c_{1}}, 1_{x}^{c_{2}} x^{c_{2}}\right\}$. Hence, the forbidden edges $U_{x}$ in $H(x)$ are given by $\bar{U}_{x}=E_{x} \backslash\left(F_{x} \cup F_{\neg x}\right)$.

- We interconnect $H(x)$ and $H(c)$, where $x$ is a literal of clause $c$, by adding edge $x_{c} x^{c}$ if $x$ appears as a positive literal and edge $x_{c} \neg x^{c}$ if $x$ appears as a negative literal. We call these edges crossing edges.

We set $U=E \backslash\left(\left(\bigcup_{c \in C} \bar{U}_{c}\right) \cup\left(\bigcup_{x \in X} \bar{U}_{x}\right)\right)$. This construction is computable within polynomial time and $G$ is a bipartite graph of maximum degree 3 . We claim that there is a truth assignment of $I$ which satisfies all clauses iff there is a maximal matching $S \subseteq U$ of $G$.

If $T$ is a truth assignment of $I$ which satisfies all clauses, then we add the set of edges $x_{c} x^{c}$ and $F_{x}$ if $T(x)=$ true; otherwise, we add the edge $x_{c} \neg x^{c}$ and all edges in $F_{\neg x}$. For each clause $c$, we choose one literal $l_{c}$ which satisfies the clause; then, we add 2 edges saturating vertices $1_{c}, 2_{c}$ and $3_{c}$ and which are not incident to the edge of $\bar{U}_{c}$ saturating $l_{c}$. For instance, assume it is $y$; then, we add two edges saturating vertices $1_{c}$ and $3_{c}$ and the white vertices in $H(c)$. The resulting matching $S$ is maximal with $S \cap \bar{U}=\emptyset$.

Conversely, assume the existence of a maximal matching $S$ with $S \subseteq U$. Hence, for each variable $x \in X$ exactly one edge between $e_{x}$ and $e_{\neg x}$ is in $S$ (in order to block edge $3_{x} 4_{x}$ ). If it is $e_{x} \in S$ (resp., $e_{\neg x} \in S$ ), then $F_{x} \subset S$ (resp., $F_{\neg x} \subset S$ ). Hence, $S$ does not contain any crossing edges saturating $\neg x^{c}$ (resp., $\left.x^{c}\right)$. Now for each clause $c=x \vee y \vee z$, at least one vertex among $x_{c}, y_{c}, z_{c}$ must be adjacent to a crossing edge of $S$. In conclusion, by setting $T(x)=$ true if at least one vertex $x^{c_{1}}$ or $x^{c_{2}}$ of $H(x)$ is saturated by $S$ and $T(x)=$ false otherwise, we get a valid assignment $T$ satisfying all clauses.

In Theorem 1, we showed that, for every fixed $r \geq 2$, EXT $r$-DCPS is hard even when the set of forbidden edges $E \backslash U$ is an induced matching. In the 
following, we prove the same result does not hold when $r=1$, by reducing this problem to the problem of finding a maximum matching in a bipartite graph.

Proposition 2. $(*)$ EXT 1-DCPS is polynomial-time decidable when the forbidden edges $\bar{U}=E \backslash U$ form an induced matching.

Remark 3. Proposition 2 can be extended to the case where $\bar{U}$ is a matching and $G_{\bar{U}}$ does not contain an alternating path of length at least 5 . The complexity of ExT 1-DCPS when $\bar{U}$ is a matching remains unsettled.

In [12], several results are proposed for the extension of the independent set problem (EXT IS for short) in bipartite graphs, planar graphs, chordal graphs, etc. Here, we deduce a new result for a subclass of claw-free graphs.

Corollary 4. ExT IS is NP-complete restricted to line graphs of bipartite graphs of maximum degree 3.

Proof. Let $G=(V, E)$ be a bipartite graph of maximum degree 3 and $L(G)=$ $\left(V^{\prime}, E^{\prime}\right)$ its line graph. It is well known that any matching $S$ of $G$ corresponds to an independent set $S^{\prime}=L(S)$ of $G^{\prime}$ and vice versa. In particular, $S$ is a maximal matching of $G$ iff $L(S)$ is a maximal independent set. Hence, $(G, U)$ is a yes-instance of ExT 1-DCPS iff $(L(G), L(U))$ is a yes-instance of ExT IS. Theorem 1 with $r=1$ concludes the proof.

A reduction from $(3, B 2)$-SAT can also be used to show the following.

Theorem 5. (*) For every fixed $r \geq 1$, ExT $r$-EC is NP-complete in bipartite graphs with maximum degree $r+2$, even if the forced edge set $U$ is a matching.

\section{Planar graphs}

All reductions given in this section are from 4-Bounded Planar 3-ConneCted SAT (4P3C3SAT for short), the restriction of EXACT 3-SATISFIABILITY ${ }^{4}$ to clauses in $\mathcal{C}$ over variables in $\mathcal{X}$, where each variable occurs in at most four clauses (at least one time but at most two times negated) and the associated bipartite graph $B P$ (explained in Section 3) is planar of maximum degree 4. This restriction is also NP-complete [23]; in the following, we always assume that the planar graph comes with an embedding in the plane. This gives us a planar variable-clause-graph $G$, corresponding to the original SAT instance $I$. The additional technical difficulties come with the embeddings that need to be preserved. Suppose that a variable $x_{i}$ appears in at most four clauses $c_{1}, c_{2}, c_{3}, c_{4}$ of $I$ such that in the induced (embedded) subgraph $G_{i}=G\left[\left\{x_{i}, c_{1}, c_{2}, c_{3}, c_{4}\right\}\right]$, $c_{1} x_{i}, c_{2} x_{i}, c_{3} x_{i}, c_{4} x_{i}$ is an anti-clockwise ordering of edges around $x_{i}$. By looking at $G_{i}$ and considering how variable $x_{i}$ appears as a negative or positive literal in the four clauses $c_{1}, c_{2}, c_{3}, c_{4}$ in $I$, the construction should handle the three following cases: (1): $x_{i} \in c_{1}, c_{2}$ and $\neg x_{i} \in c_{3}, c_{4} ;(2): x_{i} \in c_{1}, c_{3}$ and $\neg x_{i} \in c_{2}, c_{4}$; (3): $x_{i} \in c_{1}, c_{2}, c_{3}$ and $\neg x_{i} \in c_{4}$. All other cases are included in these cases by rotations and / or interchanging $x_{i}$ with $\neg x_{i}$.

${ }^{4}$ addressing the problem to decide whether there is a truth assignment setting exactly one literal in each clause to true 


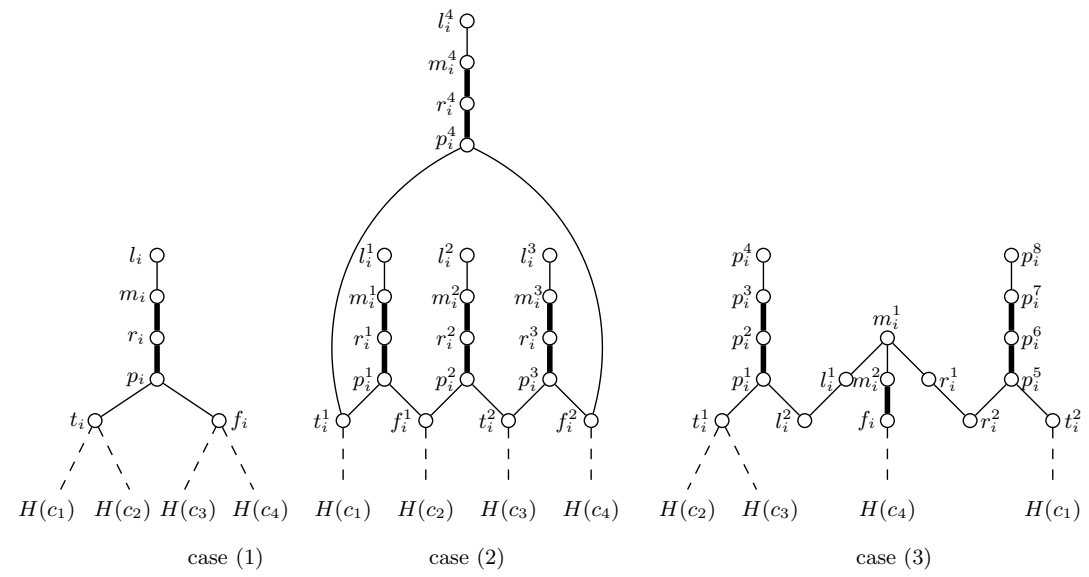

Fig. 2. Variable gadgets $H\left(x_{i}\right)$ of Theorem 6. Cases (1), (2), (3) are corresponding to $H\left(x_{i}\right)$, depending on how $x_{i}$ appears (as a negative or positive literal) in the four clauses (here, case 3 is rotated). Bold edges denote elements of $U_{x_{i}}$. Crossing edges are marked by dashed lines.

Theorem 6. For any $r \geq 1$, EXT $r$-EDS is NP-complete for planar bipartite graphs of maximum degree $r+2$.

Proof. Consider first $r=1$, corresponding to ExT EDS. Given an instance $I$ of 4P3C3SAT with clause set $\mathcal{C}=\left\{c_{1}, \ldots, c_{m}\right\}$ and variable set $\mathcal{X}=\left\{x_{1}, \ldots, x_{n}\right\}$, we build a planar bipartite graph $H=\left(V_{H}, E_{H}\right)$ with maximum degree 3 together with a set $U \subseteq E_{H}$ of forced edges as an instance of ExT EDS.

For each variable $x_{i}$ we introduce a corresponding gadget $H\left(x_{i}\right)$ as depicted in Fig. 2, the forced edge set $U_{x_{i}}$ contains $\left\{m_{i} r_{i}, r_{i} p_{i}\right\}$ for case (1), $\left\{p_{i}^{j} r_{i}^{j}, r_{i}^{j} m_{i}^{j}: 1 \leq\right.$ $j \leq 4\}$ for case (2) and $\left\{p_{i}^{1} p_{i}^{2}, p_{i}^{2} p_{i}^{3}, p_{i}^{5} p_{i}^{6}, p_{i}^{6} p_{i}^{7}, m_{i}^{2} f_{i}\right\}$ for case (3).

For each clause $c_{j} \in \mathcal{C}$, we construct a clause gadget $H\left(c_{j}\right)$ as depicted on the right, and a forced edge set (6)-(50-(40)-3c) $U_{c_{j}}$, each clause gadget $H\left(c_{j}\right)$ contains 8 vertices and 7 edges where $\left|U_{c_{j}}\right|=2$. Edges in $U$ are drawn in bold.

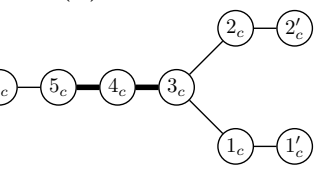

$H(c)$ for clause $c=\ell_{1} \vee \ell_{2} \vee \ell_{3}$

Moreover, we interconnect with some crossing edges the subgraphs $H\left(x_{i}\right)$ and $H\left(c_{j}\right)$ by linking $x_{i}$ (or $\neg x_{i}$ ) to $c_{j}$ according to how it appears in the clause. More precisely, each clause gadget $H\left(c_{j}\right)$ is connected to the rest of the graph via two (resp., one) crossing edges incident to $2_{c_{j}}^{\prime}$ (resp., $1_{c_{j}}^{\prime}$ ). We also set the forced edge set $U=\left(\bigcup_{x_{i} \in \mathcal{X}} U_{x_{i}}\right) \cup\left(\bigcup_{c_{j} \in \mathcal{C}} U_{c_{j}}\right)$. This construction is built in polynomial time, giving a planar bipartite graph of maximum degree 3 .

Note that by minimality, for any edge of $U$, there exist at least one private edge to dominate. So, let $S$ be a minimal edge dominating set with $S \supseteq U$, then for each clause gadget $H(c)$, at least one of the crossing edges incident to it is in $S$. Further, for each variable $x$, let $c_{t}^{x}$ (resp., $c_{f}^{x}$ ) be the set of crossing edges 
incident to $t_{i}$ (resp., $f_{i}$ ), $\left\{t_{i}^{1}, t_{i}^{2}\right\}$ (resp., $\left\{f_{i}^{1}, f_{i}^{2}\right\}$ ), and $\left\{t_{i}^{1}, t_{i}^{2}\right\}$ (resp., $f_{i}$ ) for the case 1,2 and 3 of $H(x)$ respectively, then by minimality of $S$, at most one of $\left(S \cap c_{t}^{x}\right)$ or $\left(S \cap c_{f}^{x}\right)$ is non-empty. Therefore, it can be easily checked that $I$ has a satisfying assignment $T$ iff $H$ has a minimal edge dominating set containing $U$.

For $r \geq 2$, we start with the instance $I=(H, U)$ given in the above construction for $r=1$. Recall $H=\left(V_{H}, E_{H}\right)$ is a bipartite graph with bipartition $V_{H}=L \cup R$, while $U \subseteq$ $E_{H}$ is a subset of forced edges. Now, for each vertex $v$ of the left part $L$, we add the gadget $B_{r}(v)$ depicted to the right. Denote by $H^{\prime}$ the resulting bipartite graph and consider $I^{\prime}=\left(H^{\prime}, U\right)$ as an instance of ExT $r$-EDS.

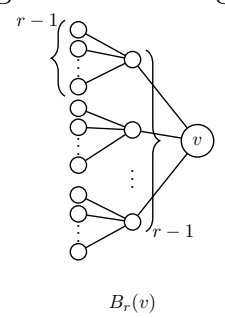

Let $B=\bigcup_{v \in L} B_{r}(v)$ be the added edges from $H$ to $H^{\prime}$. Note that any $r$-EDS $S^{\prime}$ of $H^{\prime}$ must contain $B$. Moreover, $S^{\prime}$ is a minimal $r$-EDS of $H^{\prime}$ iff $S^{\prime} \backslash B$ is a minimal EDS of $H$.

Remark \%. Reconsidering the previous construction that reduces the case when $r>1$ to the case when $r=1$, and using the NP-hardness of EDS in bipartite graphs [6,31], we deduce NP-hardness of $r$-EDS for all $r \geq 1$.

In [22], several results are proposed for the enumeration of minimal dominating sets in line-graphs. Here, we strengthen these results by showing that extending a given vertex set to a minimal dominating set (a problem we call ExT DS) in line graphs of a planar bipartite subcubic graphs is already a hard problem.

Corollary 8. EXT DS is NP-complete, even when restricted to line graphs of planar bipartite subcubic graphs.

Proof. Let $G=(V, E)$ be a bipartite graph of maximum degree 3 and $L(G)=$ $\left(V^{\prime}, E^{\prime}\right)$ its line graph. It is well known that any edge dominating set $S$ of $G$ corresponds to a dominating set $S^{\prime}=L(S)$ of $G^{\prime}$ and vice versa. In particular, $S$ is a minimal edge dominating set of $G$ iff $L(S)$ is a minimal dominating set. Hence, $(G, U)$ is a yes-instance of ExT EDS iff $(L(G), L(U))$ is a yes-instance of ExT DS. Theorem 6 with $r=1$ concludes the proof.

The two next statements appear to be only strengthening Theorems 1 and 5 in the particular case of $r=1$, but the details behind can be different indeed.

Theorem 9. (*) EXT 1-EC is NP-complete for planar bipartite subcubic graphs.

Theorem 10. (*) EXT 1-DCPS is NP-complete even for planar bipartite subcubic graphs.

\section{Parameterized perspective}

The next result is quite simple and characterizes the yes-instances of ExT $r$-EC.

Lemma 11. $(*) \operatorname{ext}(G, U) \neq \emptyset$ iff there is an $r$-EC solution $G^{\prime}=\left(V, E^{\prime}\right)$ where $E^{\prime} \supseteq U$ such that $S_{G^{\prime}}=\left\{v \in V(U): d_{G^{\prime}}(v)>r\right\}$ is an independent set of $G_{U}$. 
This structural property can be used to design an FPT-algorithm for ExT $r$-EC. More precisely, our proposed algorithm lists all $3^{|U|}$ many independent sets of $G[U]$ included in $V(U)$ from an instance $I=(G, U)$ of ExT $r$-EC. In each case, we produce an equivalent instance of MinLUCP that can be solved in polynomial time which gives the following result.

Theorem 12. (*) EXT $r$-EC, with standard parameter, is in FPT.

For ExT $r$-DCPS, we can also exploit structural properties of yes-instances and use the polynomial solvability of Simple $b$-MATCHING to show the following.

Theorem 13. $(*)$ EXT $r$-DCPS, parameterized by the number of forbidden edges $\bar{U}$, is in FPT.

When bounding the degree of the graphs, we can consider an even smaller parameter and obtain feasibility results.

Proposition 14. (*) For graphs with maximum degree $r+1$, ExT $r$-DCPS is polynomial-time decidable when $r=1$ and is in FPT with respect to the number of isolated edges in $\bar{U}$ for $r \geq 2$.

Remark 15. For graphs with maximum degree $r+1$, ExT $r$-DCPS with $r \geq 2$ is parameterized equivalent to $\mathrm{SAT}$ with respect to the number of isolated edges in $E \backslash U$ and variables, respectively.
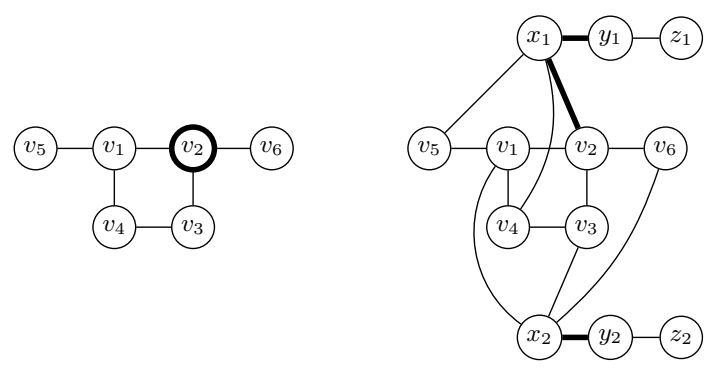

Fig. 3. $(G, U)$ as an instance of ExT VC is shown on the left, with $V_{1}=\left\{v_{2}, v_{4}, v_{5}\right\}$ and $V_{2}=\left\{v_{1}, v_{3}, v_{6}\right\}$ and $U=\left\{v_{2}\right\}$. The constructed instance $\left(G^{\prime}, U^{\prime}\right)$ of ExT EDS is shown on the right. The vertices and edges of $U$ and $U^{\prime}$ are in marked with bold lines.

Theorem 16. For any $r \geq 1$, EXT $r$-EDS (with standard parameter) is $W$ [1]hard, even when restricted to bipartite graphs.

Proof. We only consider $r=1$. For $r \geq 2$, we can use the gadget $B_{r}(v)$ as in Theorem 6. The hardness result comes from a reduction from ExT VC on bipartite graphs, the extension version of VERTEX COver; see [12]. Let $I=$ $(G, U)$ be an instance of ExT $\mathrm{VC}$, where $G=(V, E)$ is a bipartite graph with 
partition $\left(V_{1}, V_{2}\right)$ of $V$ and $U \subseteq V$, the question of VERTEx Cover is to decide if $G$ has a minimal vertex cover $S$ with $U \subseteq S$. We build an instance $I^{\prime}=\left(G^{\prime}, U^{\prime}\right)$ of ExT EDS as follows. Let us first construct a new graph $G^{\prime}=\left(V^{\prime}, E^{\prime}\right)$ with $V^{\prime}=V \cup\left\{x_{i}, y_{i}, z_{i}: i=1,2\right\}$ and

$$
E^{\prime}=E \cup \bigcup_{i=1,2}\left(\left\{x_{i} y_{i}, y_{i} z_{i}\right\} \cup\left\{v x_{i}: v \in V_{i}\right\}\right) .
$$

$G^{\prime}$ is bipartite with partition into $V_{1}^{\prime}=V_{1} \cup\left\{x_{2}, y_{1}, z_{2}\right\}$ and $V_{2}^{\prime}=V_{2} \cup\left\{x_{1}, y_{2}, z_{1}\right\}$. Set $U^{\prime}=\left\{u x_{1}: u \in U \cap V_{1}\right\} \cup\left\{u x_{2}: u \in U \cap V_{2}\right\} \cup\left\{x_{1} y_{1}, x_{2} y_{2}\right\}$ so, $\left|U^{\prime}\right|=|U|+2$. This construction is illustrated in Fig. 3 . We claim that $\left(G^{\prime}, U^{\prime}\right)$ is a yes-instance of ExT EDS if and only if $(G, U)$ is a yes-instance of Ext VC.

Suppose $(G, U)$ is a yes-instance for Ext VC; so there exists a minimal vertex cover $S$ for $G$ with $U \subseteq S$. The set $S^{\prime}=\left\{v x_{1}: v \in V_{1} \cap S\right\} \cup\left\{v x_{2}: v \in\right.$ $\left.V_{2} \cap S\right\} \cup\left\{x_{1} y_{1}, x_{2} y_{2}\right\}$ is an edge dominating set of $G^{\prime}$ which includes $U^{\prime}$ because $S$ contains $U$. Since $S$ is minimal, $S^{\prime}$ is minimal, too; observe that private edges of a vertex $v \in S \cap V_{1}$ (i.e. an edge $v u$ with $u \notin S \cap V_{1}$ ) translate to private edges of $v x_{1} \in S^{\prime}$, analogously for $x \in S \cap V_{2}$. By construction, $y_{i} z_{i}$ is a private edge for $x_{i} y_{i}, i=1,2$.

Conversely, suppose $S^{\prime}$ is a minimal edge dominating set of $G^{\prime}$ containing $U^{\prime}$. Since $S^{\prime}$ is minimal, then for each $e \in S^{\prime}$ there is a private edge set $S_{e} \subseteq E^{\prime}$, $S_{e} \neq \emptyset$, which is dominated only by $e$. Moreover, we have, for $i \in\{1,2\}$ :

$$
\forall v \in V_{i}\left(\left(v x_{i} \in S^{\prime}\right) \Longleftrightarrow\left(\forall u \in V_{3-i}\left(v u \notin S^{\prime} \cap E\right)\right)\right.
$$

since $S^{\prime}$ is minimal and $\left\{x_{1} y_{1}, x_{2} y_{2}\right\} \subseteq U^{\prime}$. We now show how to safely modify $S^{\prime}$ such that $S^{\prime} \cap E=\emptyset$. If it is not already the case, there is some edge, w.l.o.g., $e=$ $u v \in S^{\prime} \cap E$ with $u \in V_{1}$ and $v \in V_{2}$. In particular from the above observations, we deduce $u \notin U, v \notin U$ and $S_{e} \subseteq E$. Modify $S^{\prime}$ by the following procedure.

- If the private solution set $S_{e} \backslash\{e\}$ contains some edges incident to $u$ and some edges incident to $v$, then $e \in S^{\prime}$ will be replaced by $u x_{1}$ and $v x_{2}$;

- if every edge in the private solution $S_{e}$ is adjacent to $u$, replace $e$ in $S^{\prime}$ by $u x_{1}$, otherwise if every edge in the private solution $S_{e}$ is adjacent to $v$, replace $e$ in $S^{\prime}$ by $v x_{2}$.

The case distinction is necessary to guarantee that $S^{\prime}$ stays a minimal edge dominating set after each modification step. We repeat this procedure until $S^{\prime} \cap$ $E=\emptyset$. At the end of the process, every vertex $v \in V$ covers the same set of edges as $v x_{1}$ or $v x_{2}$ dominates. Hence, by setting $S=\left\{v \in V: v x_{1} \in S^{\prime}\right.$ or $\left.v x_{2} \in S^{\prime}\right\}$, we build a minimal vertex cover of $G$ containing $U$.

Remark 17. Note that the procedure of local modifications given in Theorem 16 does not preserve optimality, but only inclusion-wise minimality.

\section{Price of extension}

Considering the possibility that some set $U$ might not be extensible to any minimal solution, one might ask how far $U$ is from an extensible set. This concept, 
introduced in [11], is called Price of Extension (PoE). A similar approach has already been studied in the past called the Price of Connectivity in [10] in the context of connectivity. This notion has been introduced in [10] for MIN VC which is defined as the maximum ratio between the connected vertex cover number and the vertex cover number. Here, the goal of studying PoE is to measure how far efficiently computable extensible subsets of the given presolution $U$ are to $U$ or to the largest possible extensible subsets of $U$. To formalize this, we define optimization problems corresponding to EXT $r$-EC and EXT $r$-EDS. Actually, since we mainly propose negative results, we only focus on $r=1$ considering the problems:

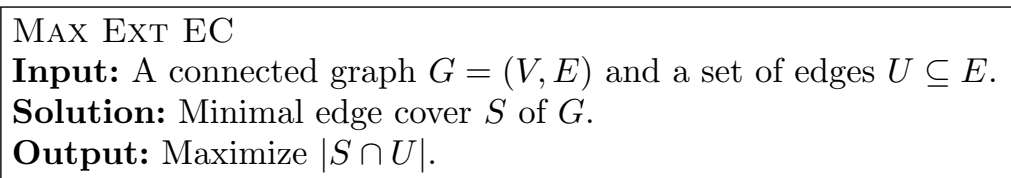

\section{MAx ExT EDS}

Input: A graph $G=(V, E)$ and a set of edges $U \subseteq E$.

Solution: Minimal edge dominating set $S$ of $G$.

Output: Maximize $|S \cap U|$.

For $\Pi=$ MAx Ext EC or $\Pi=$ Max Ext EDS, we denote the value of an optimal solution by $\operatorname{opt}_{\Pi}(G, U)$. Since for all of them $\operatorname{opt}_{\Pi}(G, U) \leq|U|$ with equality iff $(G, U)$ is a yes-instance of the extension variant, we deduce from our previous results that MAX ExT EC and MAX ExT EDS are NP-hard. In the particular case $U=E$, MAX EXT EDS is exactly the problem called UPPER EDS where the goal is to find the largest minimal edge dominating set; UPPER EDS can be also viewed as UPPER DS in line graphs. In [25], it is shown that UPPER EDS is NP-hard in bipartite graphs. Very recently, an NP-hardness proof for planar graphs of bounded degree, an APX-completeness for graphs of max degree 6 and a tight $\Omega\left(n^{\varepsilon-1 / 2}\right)$-inapproximation for general graphs and for any constant $\varepsilon \in\left(0, \frac{1}{2}\right)$, are given in [18].

The price of extension PoE is defined exactly as the ratio of approximation, i.e., $\frac{a p x}{o p t}$. We say that $\Pi$ admits a polynomial $\rho-\operatorname{PoE}$ if for every instance $(G, U)$, we can compute a solution $S$ of $G$ in polynomial time which satisfies $\operatorname{PoE}(S) \geq \rho$.

Theorem 18. For any constant $\varepsilon \in\left(0, \frac{1}{2}\right)$ and any $\rho \in \Omega\left(\Delta^{\varepsilon-1}\right)$ and $\rho \in$ $\Omega\left(n^{\varepsilon-\frac{1}{2}}\right)$, MAX ExT EC does not admit a polynomial $\rho$-PoE for general graphs of $n$ vertices and maximum degree $\Delta$, unless $\mathrm{P}=\mathrm{NP}$.

Proof. The proof is based on a reduction from the maximum independent set problem (MAX IS for short). Given a graph $G=(V, E)$ with $n$ vertices and $m$ edges where $V=\left\{v_{1}, \ldots, v_{n}\right\}$, as an instance of MAX IS, we build a connected bipartite graph $H=\left(V_{H}, E_{H}\right)$ as follows: for each $v_{i} \in V$, add a $P_{3}$ with edge set $\left\{v_{i} v_{i}^{\prime}, v_{i}^{\prime} v_{i}^{\prime \prime}\right\}$, and for each edge $e=v_{i} v_{j} \in E$ with $i<j$, add a middle vertex $v_{i, j}$ and connect $v_{i}$ to $v_{j}$ via $v_{i, j}$. Consider $I=(H, U)$ as instance of MAX EXT 

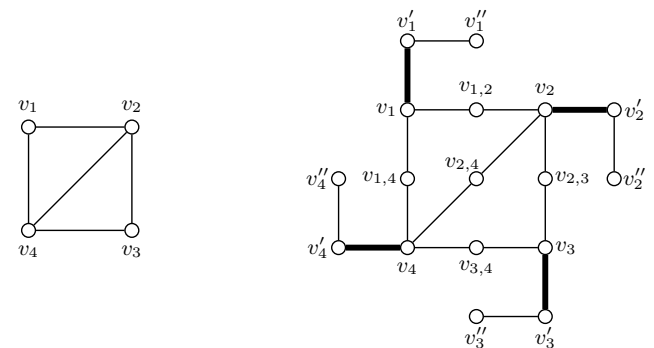

Fig. 4. On the left side, an instance of MAX IS and on the right side, the corresponding instance of MAx ExT EC. Bold edges of $H$ are the set of forced edges $U$.

EC, where the forced edge subset is given by $U=\left\{v_{i} v_{i}^{\prime}: 1 \leq i \leq n\right\}$. Clearly, $H$ is a bipartite graph with $\left|V_{H}\right|=3 n+m$ vertices, $\left|E_{H}\right|=2(m+n)$ edges and $\Delta(H)=\Delta(G)+1$. An example of this construction is illustrated in Figure 4.

We claim that there is a solution of size $k$ for Max Ext EC on $(H, U)$ iff $G$ has an independent set of size $k$. Suppose that $S$ is a maximal independent set of $G$ of size $k$. For each $e \in E$, let $v^{e} \in V \backslash S$ be a vertex which covers $e$. Clearly, $S^{\prime}=\left\{v_{i, j} v^{e}: e=v_{i} v_{j} \in E\right\} \cup\left\{v_{i}^{\prime} v_{i}^{\prime \prime}: v_{i} \in V\right\} \cup\left\{v_{i} v_{i}^{\prime}: v_{i} \in S\right\}$ is a minimal edge cover of $H$ with $\left|S^{\prime} \cap U\right|=k$. Conversely, suppose $S^{\prime}$ is a minimal edge cover of $H$ such that $\left|S^{\prime} \cap U\right|=k .\left\{v_{i}^{\prime} v_{i}^{\prime \prime}: v_{i} \in V\right\}$ is a part of every edge cover since, $v_{i}^{\prime \prime}$ for $v_{i} \in V$ are leaves of $H$. Moreover, for each $e=v_{i} v_{j} \in E$ with $i<j$, at least one edge between $v_{i} v_{i j}$ or $v_{j} v_{i, j}$ belongs to any edge cover of $H$. Furthermore, if $v_{i} v_{i, j} \in S$, by minimality we deduce that $v_{i} v_{i}^{\prime} \notin S^{\prime}$. Hence, for each $v_{i} v_{j} \in E$, at most one of $v_{i} v_{i}^{\prime}, v_{j} v_{j}^{\prime}$ can be in $S^{\prime}$. Hence, $S=\left\{v_{i}: v_{i} v_{i}^{\prime} \in S^{\prime}\right\}$ is an independent set of $G$ with size $k$.

Using the strong inapproximability results for MAX IS given in [28,32], observing $\Delta(H)=\Delta(G)+1$ and $\left|V_{H}\right| \leq 2|V|^{2}$, we obtain the claimed result.

Using result given in [18], an $\Omega\left(n^{\varepsilon-1 / 2}\right)$-inapproximation can be immediately deduced for MAx ExT EDS. The next result is obtained by a simple approximation preserving reduction from MAX EXT VC to MAX EXT EDS.

Theorem 19. $(*)$ For any constant $\varepsilon \in(0,1)$ and any $\rho \in \Omega\left(n^{\varepsilon-1}\right)$, MAX ExT EDS does not admit a polynomial $\rho$-PoE for general graphs of $n$ vertices, unless $\mathrm{P}=\mathrm{NP}$.

In contrast to the last hardness result, we give a simple approximation depending on the maximum degree $\Delta(G)$.

Theorem 20. MAX ExT EDS is $\frac{1}{\Delta\left(G_{U}\right)+1}$-approximable for instance $(G, U)$ of maximum degree $\Delta$.

Proof. Let $(G=(V, E), U)$ be an instance of Max ExT EDS, where the maximum degree of partial subgraph $G_{U}$ induced by $U$ is bounded by $\Delta$. Compute a maximum matching $M$ of $G_{U}$ and transform it into a maximal matching $M^{\prime}$ of 
$G$ containing $M$. It is well known that any maximal matching is an edge dominating set. Obviously, $\left(\Delta\left(G_{U}\right)+1\right)|M| \geq|U| \geq o p t_{\text {MAx Exт EDS }}(G, U)$ since $G_{U}$ is $\left(\Delta\left(G_{U}\right)+1\right)$-edge colorable.

Considering ExT 1-DCPS, we need to adapt the notion of the price of extension because we have to consider subset of forbidden edges (i.e., $\bar{U}$ ); more precisely, we want to increase $|U|$ as few as possible. Hence, the optimization problem called Min ExT 1-DCPS is defined as follows:

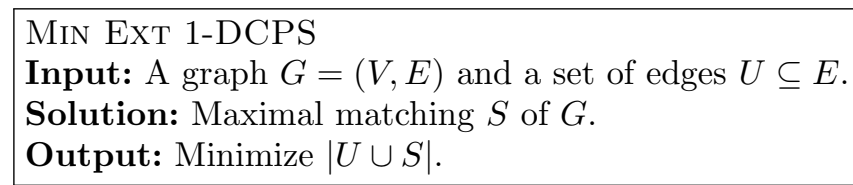

Recall that PoE is meant to measure how far efficiently computable extensible subsets are from the given presolution $U$ or to the largest possible extensible subsets of $U$. We say that Min ExT 1-DCPS admits a polynomial $\rho$-PoE if for every instance $(G, U)$, we can compute a solution $S$ of $G$ in polynomial time which satisfies $\operatorname{PoE}(S)=\frac{a p x}{o p t} \leq \rho$. In the particular case $U=\emptyset$, Min ExT 1DCPS is exactly the well known problem Minimum Maximal Matching where the goal is to find the smallest maximal matching. In $[14,15]$, it is shown that Minimum Maximal Matching is hard to approximate with a factor better than 2 and 1.18, assuming Unique Games Conjecture (UGC) and $\mathrm{P} \neq \mathrm{NP}$, respectively. We complement this bound by showing the following.

Theorem 21. $(*)$ A 2-approximation for Min ExT 1-DCPS can be computed in polynomial time.

\section{Conclusions}

We have undertaken some study on several complexity aspects of extension variants of edge graph problems. Our results should be useful in particular to the (input-sensitive) enumeration algorithms community that has so far not put that much attention on edge graph problems; we are only aware of [21] in this direction. Conversely, output-sensitive enumeration algorithms, e.g., for matchings have been around for more than twenty years [29]. Some thoughts on edge cover enumeration can be found in [30]. Our research might also inspire to revisit exact and / or parameterized algorithms on EDGE Domination; previous papers like [16] or [26] did not focus on special graph classes, where we see some potentials for future research.

\section{References}

1. Cristina Bazgan, Ljiljana Brankovic, Katrin Casel, and Henning Fernau. On the complexity ndscape of the domination chain. In Sathish Govindarajan and Anil Maheshwari, editors, CALDAM, volume 9602 of $L N C S$, pages 61-72. Springer, 2016. 
2. Cristina Bazgan, Ljiljana Brankovic, Katrin Casel, Henning Fernau, Kus Jansen, Kim-Manuel Klein, Michael mpis, Mathieu Liedloff, Jérôme Monnot, and Vangelis Th. Paschos. The many facets of upper domination. Theor. Comput. Sci., $717: 2-25,2018$.

3. André Berger, Takuro Fukunaga, Hiroshi Nagamochi, and Ojas Parekh. Approximability of the capacitated b-edge dominating set problem. Theor. Comput. Sci., 385(1-3):202-213, 2007.

4. André Berger and Ojas Parekh. Linear time algorithms for generalized edge dominating set problems. Algorithmica, 50(2):244-254, 2008.

5. Piotr Berman, Marek Karpinski, and Alex D. Scott. Approximation hardness of short symmetric instances of MAX-3SAT. ECCC, (049), 2003.

6. An A. Bertossi. Dominating sets for split and bipartite graphs. Inf. Process. Lett., 19(1):37-40, 1984.

7. Miklós Biró, Mihály Hujter, and Zsolt Tuza. Precoloring extension. I. Interval graphs. Disc. Math., 100(1-3):267-279, 1992.

8. Marthe Bonamy, Oscar Defrain, Marc Heinrich, and Jean-Florent Raymond. Enumerating Minimal Dominating Sets in Triangle-Free Graphs. In Rolf Niedermeier and Christophe Paul, editors, STACS, volume 126 of Leibniz International Proceedings in Informatics (LIPIcs), pages 16:1-16:12, Dagstuhl, Germany, 2019. Schloss Dagstuhl-Leibniz-Zentrum fuer Informatik.

9. Endre Boros, Vdimir Gurvich, and Peter L. Hammer. Dual subimplicants of positive Boolean functions. Optim. Meth. Softw., 10(2):147-156, 1998.

10. Jean Cardinal and Eythan Levy. Connected vertex covers in dense graphs. Theor. Comput. Sci., 411(26-28):2581-2590, 2010.

11. Katrin Casel, Henning Fernau, Mehdi Khosravian Ghadikoei, Jérôme Monnot, and Florian Sikora. On the complexity of solution extension of optimization problems. CoRR, abs/1810.04553, 2018.

12. Katrin Casel, Henning Fernau, Mehdi Khosravian Ghadikoei, Jérôme Monnot, and Florian Sikora. Extension of vertex cover and independent set in some classes of graphs. In Pinar Heggernes, editor, CIAC, volume 11485 of LNCS. Springer, 2019.

13. Charles J. Colbourn. The complexity of completing partial Latin squares. Disc. Appl. Math., 8(1):25-30, 1984.

14. Szymon Dudycz, Mateusz Lewandowski, and Jan Marcinkowski. Tight approximation ratio for minimum maximal matching. In Andrea Lodi and Viswanath Nagarajan, editors, IPCO, volume 11480 of LNCS, pages 181-193. Springer, 2019.

15. Bruno Escoffier, Jérôme Monnot, Vangelis Th. Paschos, and Mingyu Xiao. New results on polynomial inapproximabilityand fixed parameter approximability of edge dominating set. Theory of Computing Systems, 56(2):330-346, 2015.

16. Henning Fernau. Edge dominating set: Efficient enumeration-based exact algorithms. In Hans L. Bodender and Michael A. Langston, editors, IWPEC, volume 4169 of $L N C S$, pages 142-153. Springer, 2006.

17. Henning Fernau and Stefan Hoffmann. Extensions to minimal synchronizing words. Journal of Automata, nguages and Combinatorics, 24, 2019.

18. Henning Fernau, David F. Manlove, and Jérôme Monnot. Algorithmic study of upper edge dominating set. Manuscript, 2019.

19. Harold N. Gabow. An efficient reduction technique for degree-constrained subgraph and bidirected network flow problems. In David S. Johnson, Ronald Fagin, Michael L. Fredman, David Harel, Richard M. Karp, Nancy A. Lynch, Christos H. Papadimitriou, Ronald L. Rivest, Walter L. Ruzzo, and Joel I. Seiferas, editors, STOC, pages 448-456. ACM, 1983.

20. Michael R. Garey and David S. Johnson. Computers and Intractability: A Guide to the Theory of NP-Completeness. W. H. Freeman \& Co., 1979. 
21. Petr A. Golovach, Pinar Heggernes, Dieter Kratsch, and Yngve Vilnger. An incremental polynomial time algorithm to enumerate all minimal edge dominating sets. Algorithmica, 72(3):836-859, 2015.

22. Mamadou Moustapha Kanté, Vincent Limouzy, Arnaud Mary, and Lhouari Nourine. On the neighbourhood helly of some graph csses and applications to the enumeration of minimal dominating sets. In Kun-Mao Chao, Tsan-sheng Hsu, and Der-Tsai Lee, editors, ISAAC, volume 7676 of $L N C S$, pages 289-298. Springer, 2012.

23. Jan Kratochvíl. A special pnar satisfiability problem and a consequence of its NP-completeness. Disc. Appl. Math., 52:233-252, 1994.

24. Eugene L. Lawler, Jan Karel Lenstra, and A. H. G. Rinnooy Kan. Generating all maximal independent sets: NP-hardness and polynomial-time algorithms. SIAM J. Comp., 9:558-565, 1980.

25. Alice A. McRae. Generalizing NP-completeness proofs for bipartite and chordal graphs. PhD thesis, Clemson University, Department of Computer Science, South Carolina, 1994.

26. Johan M. M. van Rooij and Hans L. Bodlaender. Exact algorithms for edge domination. Algorithmica, 64(4):535-563, 2012.

27. Alexander Schrijver. Combinatorial Optimization: Polyhedra and Efficiency. Springer-Verg, 2003.

28. Luca Trevisan. Non-approximability results for optimization problems on bounded degree instances. In Jeffrey Scott Vitter, Paul G. Spirakis, and Mihalis Yannakakis, editors, STOC, pages 453-461. ACM, 2001.

29. Takeaki Uno. Algorithms for enumerating all perfect, maximum and maximal matchings in bipartite graphs. In Hon Wai Leong, Hiroshi Imai, and Sanjay Jain, editors, ISAAC, volume 1350 of $L N C S$, pages 92-101. Springer, 1997.

30. Jianxin Wang, Beiwei Chen, Qilong Feng, and Jianer Chen. An efficient fixedparameter enumeration algorithm for weighted edge dominating set. In Xiaotie Deng, John E. Hopcroft, and Jinyun Xue, editors, FAW, volume 5598 of LNCS, pages 237-250. Springer, 2009.

31. Mihalis Yannakakis and Fanica Gavril. Edge dominating sets in graphs. SIAM Journal on Applied Mathematics, 38(3):364-372, 1980.

32. David Zuckerman. Linear degree extractors and the inapproximability of max clique and chromatic number. Theory of Computing, 3(1):103-128, 2007. 